\title{
Circumstellar environment of 55 Cancri
}

\section{The super-Earth 55 Cnce as a primary target for star-planet interactions *}

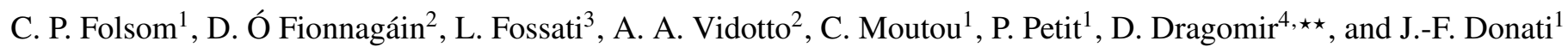 \\ ${ }^{1}$ IRAP, Université de Toulouse, CNRS, UPS, CNES, 31400 Toulouse, France \\ e-mail: cfolsom@irap.omp.eu \\ 2 Trinity College Dublin, College Green, Dublin 2, Ireland \\ ${ }^{3}$ Space Research Institute, Austrian Academy of Sciences, Schmiedlstrasse 6, 8042 Graz, Austria \\ e-mail: luca.fossati@oeaw.ac.at \\ ${ }^{4}$ Department of Physics and Kavli Institute for Astrophysics and Space Research, MIT, Cambridge, MA 02139, USA
}

Received 25 November 2019 / Accepted 8 December 2019

\begin{abstract}
Context. 55 Cancri hosts five known exoplanets, most notably the hot super-Earth $55 \mathrm{Cnc}$, which is one of the hottest known transiting super-Earths.

Aims. Because of the short orbital separation and host star brightness, 55 Cnc e provides one of the best opportunities for studying star-planet interactions (SPIs). We aim to understand possible SPIs in this system, which requires a detailed understanding of the stellar magnetic field and wind impinging on the planet.

Methods. Using spectropolarimetric observations and Zeeman Doppler Imaging, we derived a map of the large-scale stellar magnetic field. We then simulated the stellar wind starting from the magnetic field map, using a 3D magneto-hydrodynamic model.

Results. The map of the large-scale stellar magnetic field we derive has an average strength of $3.4 \mathrm{G}$. The field has a mostly dipolar geometry; the dipole is tilted by $90^{\circ}$ with respect to the rotation axis and the dipolar strength is $5.8 \mathrm{G}$ at the magnetic pole. The wind simulations based on this magnetic geometry lead us to conclude that $55 \mathrm{Cnc}$ e orbits inside the Alfvén surface of the stellar wind, implying that effects from the planet on the wind can propagate back to the stellar surface and result in SPI.
\end{abstract}

Key words. stars: individual: $55 \mathrm{Cnc}$ - stars: magnetic field - stars: late-type - stars: winds, outflows - planetary systems planet-star interactions

\section{Introduction}

The 55 Cnc system is one of the most relevant systems for understanding planets with masses or radii in between those of Earth and Neptune. This mass-radius regime, which is characterised by a large variety of bulk densities, is not found in the solar system, but yet constitutes the largest population of known exoplanets.

$55 \mathrm{Cnc}(\mathrm{G} 8 \mathrm{~V})$ hosts five known exoplanets, most notably the close-in super-Earth $55 \mathrm{Cnc}$ e $\left(M_{\mathrm{p}}=8.37 \pm 0.38 M_{\oplus}, R_{\mathrm{p}}=1.92 \pm\right.$ $\left.0.08 R_{\oplus}, P_{\text {orb }}=18 \mathrm{~h}\right)$. This exoplanet offers one of the best opportunities for detailed characterisation of a super-Earth thanks to two key properties: at $V \approx 6$, the host star is the third brightest star known to host a transiting planet; and because of the high planetary temperature, there is a high planet-star flux contrast, making the planetary emission well detectable at optical and infrared (IR) wavelengths.

Observations suggest that $55 \mathrm{Cnc}$ and $55 \mathrm{Cnc}$ e (also called "planet e") may be an up-scaled analogue of the Jupiter-Io system (Demory et al. 2016a). This would imply the presence of a significant exosphere surrounding the planet and plasma flowing from the planet towards the star, following the stellar magnetic field lines. Spitzer Space Telescope observations conducted at $4.5 \mu \mathrm{m}$ indicate the dayside thermal emission of the planet varied

\footnotetext{
* Based on observations obtained at the Telescope Bernard Lyot (USR5026) operated by the Observatoire Midi-Pyrénées, Université de Toulouse (Paul Sabatier), Centre National de la Recherche Scientifique of France.

${ }^{\star \star}$ Hubble Fellow.
}

by about 300\% between 2012 and 2013; temperatures ranged between 1300 and $3000 \mathrm{~K}$ (Demory et al. 2016b). This can be explained assuming that the planetary lithosphere is partially molten, particularly on the dayside.

The Microvariability and Oscillations of Stars telescope (MOST) satellite observations (Winn et al. 2011; Dragomir et al. 2014) show variability in the visible that could be linked to the IR variability. The MOST phase curve in 2012 shows a smaller amplitude than in 2011, and the former is comparable to the IR phase curve. This has led to suggestions that there is opaque material in the visible and IR, such as grains of dust coupled to hot plasma, similar to Io (Krüger et al. 2003). The grey behaviour of dust could provide the same opacity both in the visible and IR.

More recently, Ridden-Harper et al. (2016) detected absorption at the position of the CaII H\&K resonance lines, which may be connected with the exosphere of planet e. This $4.9 \sigma$ detection was achieved only for one of the four datasets, suggesting temporal variability in the optical depth of the material surrounding the planet.

Reconciling these properties would require the presence of azimuthally inhomogeneous circumstellar material and/or of a large exosphere made of ions and charged dust particles similar to Io (Krüger et al. 2003), which could contribute to a variable grey opacity along the line of sight through diffusion of stellar light. The large-scale structure of the circumstellar material appears to remain stable for days or weeks (Demory et al. 2016b), which would likely require a large-scale stellar magnetic field for support. 
A large stellar magnetic field would also be able to connect the star and planet, creating chromospheric hotspots. Again, we find an analogy with the Io-Jupiter system, in which observations indicate the existence of auroral hotspots in Jupiter at the footpoints of magnetic field lines connecting Io to Jupiter (Bhardwaj \& Gladstone 2000). Therefore, detecting the presence of a significant magnetic field on $55 \mathrm{Cnc}$ would support the idea that this system is a scaled-up version of the Jupiter-Io system. Examining the stellar magnetic and wind properties would also clarify the likelihood of magnetic star-planet interactions (SPIs).

We present the detection and characterisation of the stellar surface magnetic field of $55 \mathrm{Cnc}$ and model its stellar wind. This paper follows an earlier unsuccessful search for a magnetic field for 55 Cnc by Marcy (1984) using Zeeman broadening. From our simulations, we propose that $55 \mathrm{Cnc}$ e should be a primary target for detecting SPIs, and instrumentation available in the near future may indeed lead to such a detection.

\section{Zeeman Doppler imaging}

To detect and characterise the magnetic field of $55 \mathrm{Cnc}$, we observed the star using the Narval spectropolarimeter at the Pic du Midi Observatory in France. Narval includes a polarimeter module connected by fibre to a high resolution $(R \sim 65000)$ échelle spectrograph (3700-10500 ̊). Observations were made in Stokes $V$ mode, providing both total intensity (Stokes $I$ ) and circularly polarised (Stokes $V$ ) spectra. Data were reduced using the LIBREESPRIT pipeline (Donati et al. 1997) in the version built for Narval.

We obtained 20 observations of $55 \mathrm{Cnc}$ on ten nights between March 1 and April 21, 2017. Two consecutive observations were obtained on each night to allow the possibility of co-adding observations. Magnetic detections were obtained reliably in single observations, therefore co-adding was not needed. A total exposure time of $3600 \mathrm{~s}$ (as a sequence of four $900 \mathrm{~s}$ sub-exposures) was used.

To detect Zeeman splitting in Stokes $V$, we used least squares deconvolution (LSD; Donati et al. 1997; Kochukhov et al. 2010). This is a cross-correlation technique that produces pseudoaverage line profiles with greatly reduced noise. We used the line mask for a $5000 \mathrm{~K}$ star from Folsom et al. (2018a), a normalising wavelength of $650 \mathrm{~nm}$, a normalising Landé factor of 1.195, and the code of Donati et al. (1997). We obtained magnetic detections in the $V$ LSD profiles (Fig. 1) on all nights except for April 10, and those non-detections appear to be due to the rotation phase of the star.

We characterised the magnetic field strength and geometry of $55 \mathrm{Cnc}$ using Zeeman Doppler imaging (ZDI; e.g. Donati et al. 2006). The ZDI technique uses a regularised fitting procedure to model the rotationally modulated Stokes $V$ LSD profiles and infer the simplest magnetic geometry needed to reproduce the profiles. We used the ZDI code of Folsom et al. (2018a), following the analysis procedure of Folsom et al. (2018b). The $v \sin i$ of $55 \mathrm{Cnc}$ is poorly constrained since it is below the resolution of Narval. We inferred an equatorial velocity of $1.24 \mathrm{~km} \mathrm{~s}^{-1}$, using the interferometric radius of $0.943 \pm 0.010 R_{\odot}$ from von Braun et al. (2011; confirmed by Ligi et al. 2016), and the rotation period of $38.8 \pm 0.05 \mathrm{~d}$ from Bourrier et al. (2018), which is within $3 \sigma$ of $40.7 \pm 0.7 \mathrm{~d}$ from Hempelmann et al. 2016. To constrain the intrinsic line profile shape we fit the line in Stokes $I$ with a Voigt profile, broadened by this upper limit on $v \sin i$, and found a Gaussian width of $2.32 \mathrm{~km} \mathrm{~s}^{-1}$ and a Lorentzian width of $0.81\left(2.84 \mathrm{~km} \mathrm{~s}^{-1}\right)$ (cf. Folsom et al. 2018b).

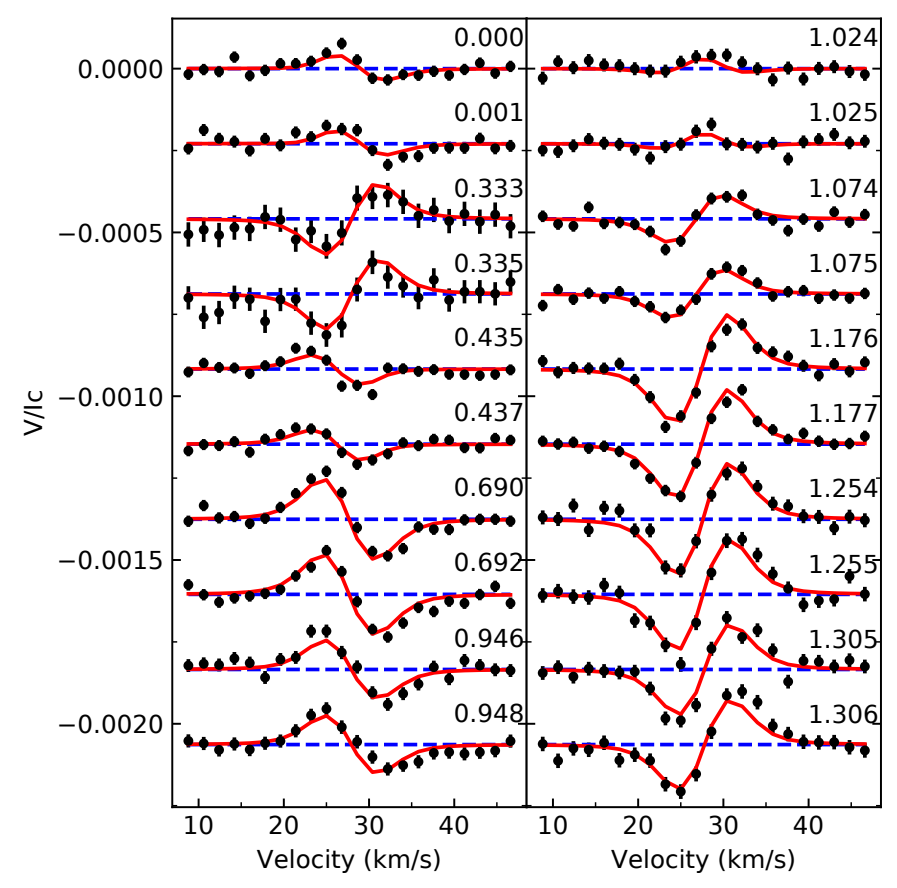

Fig. 1. Stokes $V$ profiles via LSD of 55 Cnc (black points), labelled by rotation cycle for a 39-day period shifted vertically for clarity. The dashed lines indicate zero and solid lines are the best-fit ZDI model profiles.

Using the above equatorial velocity and intrinsic line widths, we constrained the inclination of the stellar rotation axis $(i)$ via ZDI to fit the Stokes $V$ profile variability. This was done by fitting ZDI models with a wide range of inclinations and looking for values that provided the best fit. We find $i>80^{\circ}$ with a $1 \sigma$ confidence (and $i>65^{\circ}$ at a $3 \sigma$ confidence), with the most likely values near $90^{\circ}$. Bourrier et al. (2018) find an orbital inclination for $55 \mathrm{Cnc}$ e of $83.59_{-0.44}^{+0.47 \circ}$, thus our rotation inclination is consistent with the stellar rotation axis and planet orbital axis being aligned.

We re-derived the rotation period using ZDI fitting of the LSD profiles, as was done by Folsom et al. (2018b) and found $39.0 \pm 0.3 \mathrm{~d}$, which is in good agreement with Bourrier et al. (2018). We searched for surface differential rotation via ZDI, as in Folsom et al. (2018b), but find no strong evidence for it. We find that $\mathrm{d} \Omega$ (the difference in angular frequency between the pole and equator) is $<0.065 \mathrm{rad} \mathrm{day}^{-1}$ at a $3 \sigma$ confidence level, and that this parameter covaries strongly with rotation period. The uncertainty is based on variations in $\chi^{2}$ for models computed with different period and $\mathrm{d} \Omega$ but the same entropy, as in Folsom et al. (2018b). This result implies that differential rotation, if at all present, is not anomalously strong in this star compared to other stars of this spectral type. The specific value of $d \Omega$ within this uncertainty range has a minimal impact on the magnetic map, changing field strengths and how poloidal or axisymmetric the field is by less than $2 \%$. Our data span only 1.3 rotation cycles, further complicated by the low $v \sin i$, which likely explains the lack of evidence for differential rotation in the data.

The final magnetic map is presented in Fig. 2 and corresponding line profile fits are in Fig. 1 for $i=90^{\circ}, P=39.0 \mathrm{~d}$, and $\mathrm{d} \Omega=0 \mathrm{rad} \mathrm{d}^{-1}$. We find an average unsigned large-scale field of $3.4 \mathrm{G}$. The large-scale field we reconstruct is dominantly poloidal $\left(99 \%\right.$ of the magnetic energy, as calculated from $B^{2}$ ), and most of that poloidal field is in the dipole ( $79 \%$ energy) and quadrupole (19\% energy) components. The strength of the 

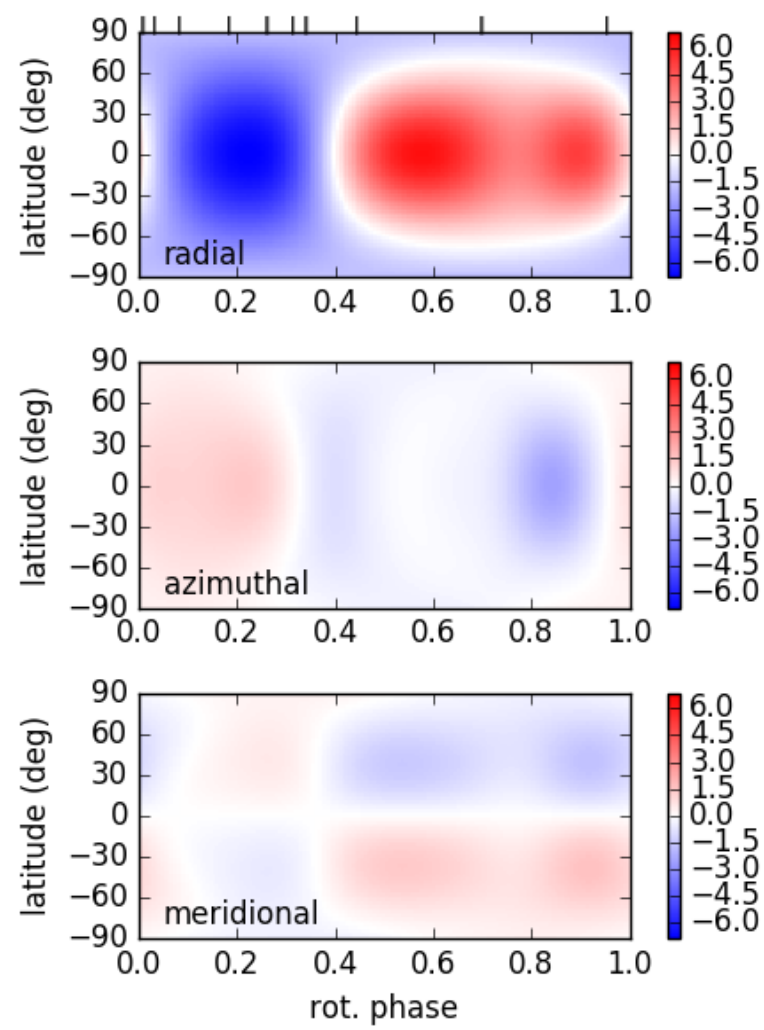

Fig. 2. Magnetic map of 55 Cnc from ZDI. The radial, azimuthal, and meridional components of the magnetic field are presented in units of G. The ticks above the top panel indicate phases in which observations were obtained.

dipolar component, at the magnetic pole, is $5.8 \mathrm{G}$. The magnetic field we find is dominantly non-axisymmetric ( $94 \%$ energy, $m \neq 0$ ), although that may be biased because the stellar inclination is near $90^{\circ}$.

To investigate the impact of an unexpectedly large error in the inclination, we computed a magnetic map with $i=60^{\circ}$, and found the map was largely unchanged. The axisymmetry of the poloidal component is virtually unchanged, while the dipolar axisymmetry increases by $5 \%$. We derive the same poloidal fraction, although $4 \%$ of the poloidal energy shifts from the dipole to the quadrupole and octopole components.

As the inclination approaches $90^{\circ}, \mathrm{ZDI}$ (like regular Doppler imaging) suffers from north-south "mirroring" effects. More precisely, in terms of spherical harmonics, components where $l+m$ is an odd number, i.e. where $B(\theta, \phi)=-B(\pi-\theta, \phi)$, are not constrained when $i=90^{\circ}$ and thus are forced to zero by the regularisation. Most notably that includes the axisymmetric dipole component $(l=1, m=0)$, therefore there is likely some magnetic field to which our observations are not sensitive. The phase sampling of the observations between 0.5 and 1.0 is relatively coarse, which likely lowers the resolution of the magnetic map in that hemisphere. However, for the stellar wind near planet $\mathrm{e}$ and beyond, only the most large-scale features (lowest degree spherical harmonic) dominate (e.g. Jardine et al. 2017).

\section{Stellar wind around 55 Cnc}

We used the numerical modelling tool BATS-R-US (Powell et al. 1999) to simulate the stellar wind of $55 \mathrm{Cnc}$. This code has been used to simulate astrophysical plasma environments (Tóth et al. 2005; Manchester et al. 2008; Vidotto et al. 2015;

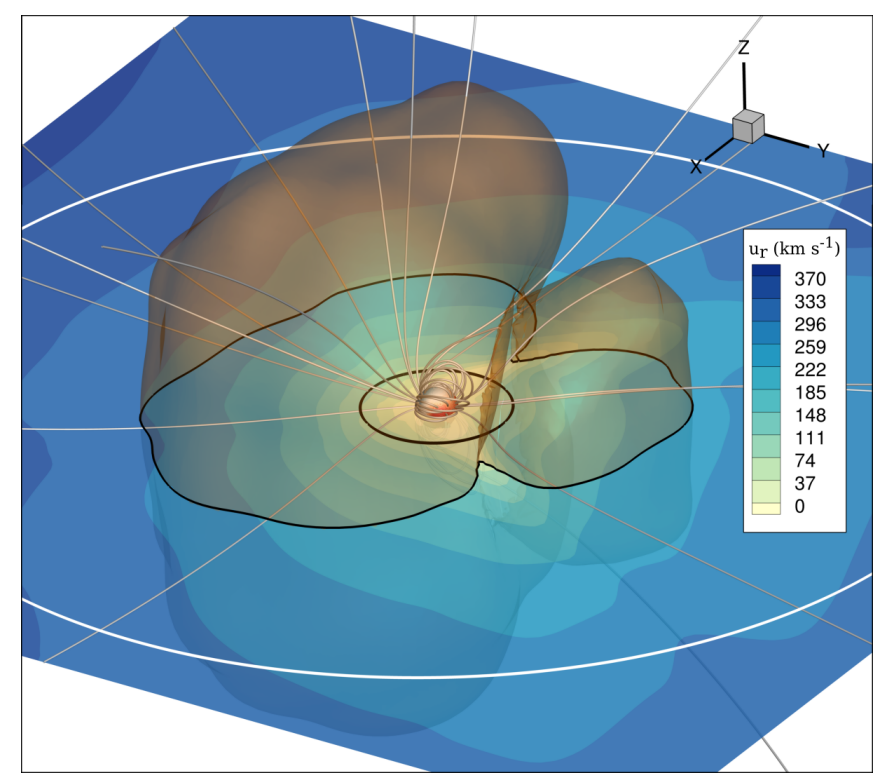

Fig. 3. Three-dimensional MHD simulation of $55 \mathrm{Cnc}$. Wind velocity is shown as a slice at the $z=0$ plane and extends to $30 R_{\star}$. The Alfvén surface is shown in orange; its intersection with the orbital plane of the planets is highlighted with a thick black line. Open magnetic field lines are shown in grey and closed magnetic field lines are shown in red. The orbits of $55 \mathrm{Cnc} \mathrm{e}$ and $\mathrm{b}$ are represented in black and white respectively.

Alvarado-Gómez et al. 2018), and in this work we used the version from Vidotto et al. (2015, more details can be found in that paper). The BATS-R-US tool solves the set of closed, ideal magnetohydrodynamic (MHD) equations for mass, momentum, and energy conservation, and the magnetic induction equation. The code solves for eight parameters: mass density $(\rho)$, velocity $\left(\boldsymbol{u}=\left\{u_{x}, u_{y}, u_{z}\right\}\right)$, magnetic field $\left(\boldsymbol{B}=\left\{B_{x}, B_{y}, B_{z}\right\}\right)$, and gas pressure $(P)$. We assume that the plasma behaves as an ideal gas, hence $P=n k_{\mathrm{B}} T$, where $n=\rho /\left(\mu m_{\mathrm{p}}\right)$ is the total number density of the wind, where $\mu m_{\mathrm{p}}$ denotes the average particle mass. We take $\mu=0.5$, which represents a fully ionised hydrogen wind. Pressure is related to density in the wind by the polytropic relation: $P \propto \rho^{\gamma}$, where $\gamma$ is the polytropic index. Through the polytropic index, heating is implicitly added to the wind. We adopt $\gamma=1.05$, similar to values used in the literature (Vidotto et al. 2015; Réville et al. 2015; Ó Fionnagáin \& Vidotto 2018). Including the polytropic index, this model contains three free parameters; the remaining two are the base density $\left(\rho_{0}\right)$ and base temperature $\left(T_{0}\right)$ of the wind. For this simulation we used $\rho_{0}=10^{-16} \mathrm{~g} \mathrm{~cm}^{-3}$ and $T_{0}=1 \mathrm{MK}$. We simulated the stellar wind in a grid that extends to $30 R_{\star}$ in each direction. Our simulation has a range of resolutions from $0.019-0.625 R_{\star}$. We find that $55 \mathrm{Cnc}$ is losing mass at a rate of $2.2 \times 10^{-14} M_{\odot} \mathrm{yr}^{-1}$, similar to the solar mass-loss rate of $2 \times 10^{-14} M_{\odot} \mathrm{yr}^{-1}$, and angular momentum at a rate of $8.2 \times 10^{29} \mathrm{erg}$.

Figure 3 shows the output of our simulation, where the black and white circles represent the orbits of planets e and b at 3.5 and $26.2 R_{\star}$, respectively. Extracting values from the wind at these orbits allows us to investigate the environment surrounding these planets. Table 1 shows the values of the stellar wind properties averaged over one planetary orbit for each of the known planets. As some of the planets are outside the domain of our simulation, we perform an extrapolation of the wind values at planets $c, f$, and $\mathrm{d}$. While planet e is impacted by a much slower wind, the wind still imparts a higher ram pressure upon planet e than upon 
Table 1. Stellar wind local properties around the known $55 \mathrm{Cnc}$ planets averaged over one planetary orbit.

\begin{tabular}{lcccccc}
\hline \hline Orbital wind properties & "e" & "b" & "c" & "f" & "d" & Reference \\
\hline Orbital period (days) & 0.74 & 14.7 & 44.4 & 262 & 4825 & Baluev (2015) \\
Semi-major axis (au) & 0.0154 & 0.1134 & 0.2373 & 0.7708 & 5.957 & Bourrier et al. (2018) \\
Semi-major axis $\left(R_{\star}\right)$ & 3.52 & 25.85 & 54.10 & 175.74 & 1358.17 & Bourrier et al. (2018) \\
Stellar wind density $\left(\mathrm{g} \mathrm{cm}^{-3}\right)$ & $4.5 \times 10^{-19}$ & $7.7 \times 10^{-22}$ & $1.4 \times 10^{-22}$ & $1.2 \times 10^{-23}$ & $1.7 \times 10^{-25}$ & This work \\
Velocity $\left(\mathrm{km} \mathrm{s}^{-1}\right)$ & 87.5 & 343.0 & 384.5 & 384.5 & 384.5 & This work \\
Velocity incl. orbital motion $\left(\mathrm{km} \mathrm{s}^{-1}\right)$ & 244.1 & 349.4 & 388.9 & 385.9 & 384.6 & This work \\
Ram pressure $\left(10^{-7}\right.$ dyn cm $\left.{ }^{-2}\right)$ & 313.1 & 8.69 & 2.06 & 0.27 & 0.008 & This work \\
Temperature $\left(10^{6} \mathrm{~K}\right)$ & 1.08 & 0.837 & 0.761 & 0.666 & 0.528 & This work \\
Magnetic field $(\mathrm{nT})$ & 5021 & 39.02 & 7.04 & 0.56 & 0.007 & This work \\
\hline
\end{tabular}

planet $b$, owing to the much higher wind density closer to the star.

Figure 3 shows that planet e orbits entirely within the Alfvén surface of the wind of $55 \mathrm{Cnc}$. This means that the wind is magnetically dominated around this planet. Being in a sub-Alfvénic regime means that planet e has a direct connection to the star; in the sub-Alfvénic regime, the planet can generate Alfvén wings, which can couple to the star and carry electromagnetic energy towards the star (e.g. Saur et al. 2013; Fischer \& Saur 2019). This scenario has ramifications for the SPI, as we discuss in the next section. An important factor to keep in mind is that the size of the Alfvén surface depends on the stellar magnetic field and wind base density. Although the stellar magnetic field is tied to the observations, the density is a free parameter in our model. Thus, we performed two additional sets of simulations, with three times larger and three times smaller base density. These choices affect, for example, the mass-loss rate of the stellar wind, but in all three scenarios we explored, the orbit of planet e remains entirely within the Alfvén surface.

\section{Star-planet interactions induced by planet 55 Cnc e}

It has been suggested that close-in planets could enhance stellar activity via SPI, which would be able to generate inducedchromospheric hotspots in the host star (Cuntz et al. 2000; Cuntz \& Shkolnik 2002). Potential signatures of SPI in the form of anomalous chromospheric emission modulated by a planet has been reported for a few systems, such as in the case of HD 179949 (Shkolnik et al. 2008; Fares et al. 2012) and HD 189733 (Cauley et al. 2018). However, not all close-in planets can generate induced emission in their host stars (Shkolnik et al. 2005, 2008; Miller et al. 2012, 2015). One possible suggestion is that if the planet orbits beyond the Alfvén surface, information from a potential star-planet magnetic reconnection event would be prevented from propagating towards the star. Given the sub-Alfvénic orbit of $55 \mathrm{Cnc} \mathrm{e}$, we investigate in this work the potential sites of generation of chromospheric hotspots caused by SPI. Figure 4 shows a set of 100 magnetic field lines that intercept planet e as it orbits around the star. If chromospheric hotspots can be formed as a result of "magnetic SPI" (i.e. through magnetic reconnection or Alfvén wings; Neubauer 1980, Ip et al. 2004), the footpoints of the connecting lines would tell us where and when such hotspots would appear.

Figure 4 shows that the magnetic field lines linking the planet to the star are a combination of open lines and closed loops. These closed magnetic loops cause the SPI-related

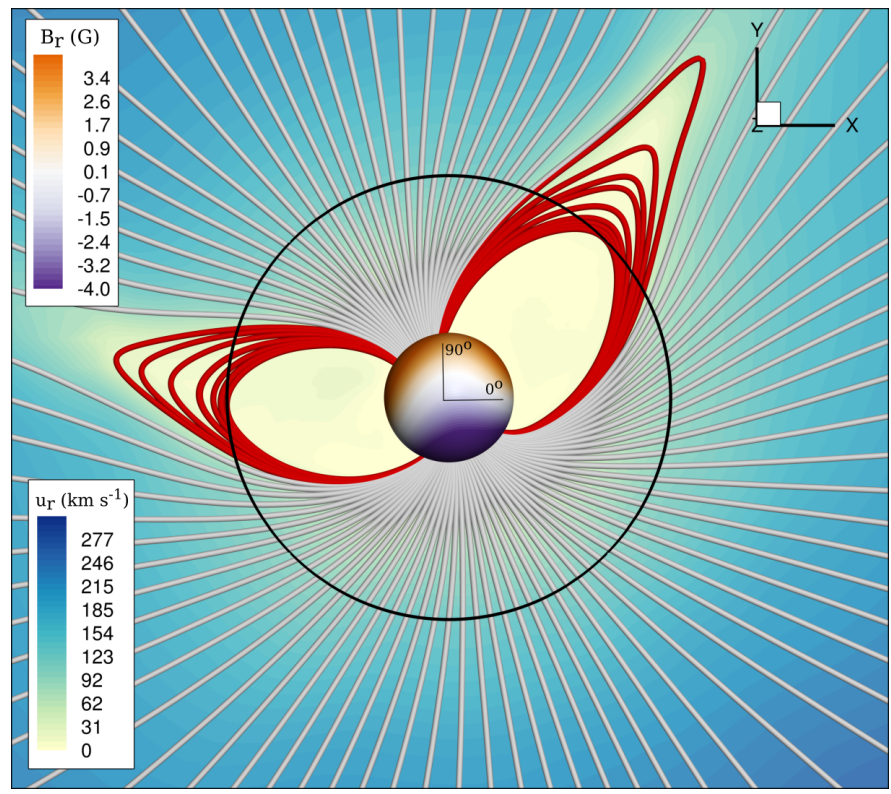

Fig. 4. Stellar magnetic field lines that intercept planet e as it orbits around the star (black circle). Stellar rotation axis is perpendicular to this orbital plane. The red field lines correspond to closed field regions. The top colour bar shows magnetic field strength (purple-orange) and bottom colour bar shows the stellar wind velocity (yellow-blue).

chromospheric spots on the star to move differently from the planetary orbit; large jumps occur where the planet moves from one branch of the closed loop to another. This phase lag and jumping effect is evident from Fig. 5 (see also predictions by McIvor et al. 2006). In fact, Fig. 5 shows that the footpoints move from lagging behind the planet orbit to ahead of it. Furthermore, for the closed loops, it is expected that the SPI occurs at both footpoints of the loop, unless that loop extends beyond the Alfvén surface. Recently, Strugarek et al. (2019) show a similar effect in the case of Kepler-78, where the magnetic topology of the host star can greatly affect the transient nature of SPI. Although their simulations do not explain the amplitude of enhanced activity observed by Moutou et al. (2016), in stars with stronger magnetic fields (e.g. HD 179949; Fares et al. 2012) the effect may be more detectable.

As the observed magnetic field of $55 \mathrm{Cnc}$ is largely described by a dipole tilted by $90^{\circ}$ to the rotation axis, all of the magnetic field lines that intersect with the planetary orbit lie within the equatorial plane. This is specific to the case of $55 \mathrm{Cnc}$ and to the epoch of ZDI observation. For other planetary systems and/or 

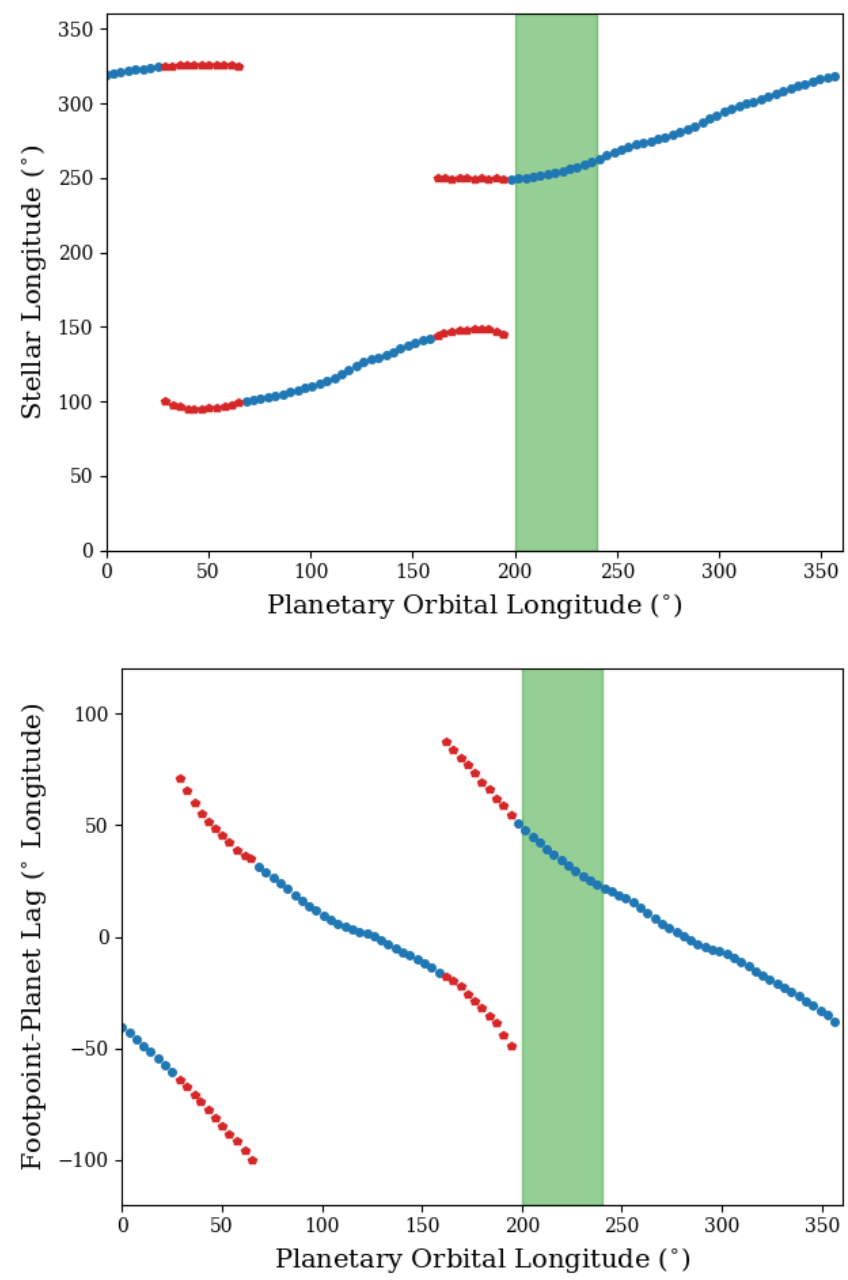

Fig. 5. Top: longitude of the footpoint on the stellar surface vs. the longitude of the planetary orbit, as defined in Fig. 4. Footpoints of open (closed) magnetic field lines are shown in blue (red). Bottom: phase lag (difference in longitudes between footpoint and subplanetary point) vs. the orbital longitude of the planet. The green region indicates the location during planetary transit.

different observing epochs, the magnetic field footpoints may lie above/below the equator, and this is dependent on the large-scale stellar magnetic field geometry. The magnetic field is essential for identifying the theoretical period of modulation for magnetic SPI. An aligned dipole generates a modulation at the synodic period $\left(0.73 \mathrm{~d}\right.$ for $55 \mathrm{Cnc}$ e), while a dipole tilted at $90^{\circ}$ generates a modulation of half the synodic period. The visibility of magnetic footpoints are also modulated by the stellar rotation period. For $55 \mathrm{Cnc}$, because the field is not purely dipolar, the distribution of footpoints on the stellar surface is not symmetric about the rotation axis; the branch of footpoints of positive polarity (Fig. 4) occupies a smaller range of stellar longitudes than that of negative polarities. This can also be seen in the top panel of Fig. 5, in which we see that the branch of footpoints above $180^{\circ}$ of stellar longitude lasts longer than the footpoints below $180^{\circ}$. An additional phase lag between the planet and induced stellar activity may be present as a consequence of the time it takes the Alfvén waves to travel to the star (Strugarek et al. 2019). For $55 \mathrm{Cnc}$, however, this travel time lag is less than $10 \%$ of the orbital period.

Because the inclination of the rotation axis of $55 \mathrm{Cnc}$ is near $90^{\circ}$, we cannot strongly constrain the axisymmetric dipolar component of the magnetic field. Unfortunately, this limitation is intrinsic to any Stokes $V$ observations and additional observations will not simply resolve it. Depending on how strong an unseen $m=0$ dipole is, this could change the position of hotspots on the surface of the star, mostly in latitude, as the connectivity between the stellar magnetic field and planet could change. In terms of wind modelling, adding a dipolar component with $m=0$ to the magnetic map of $55 \mathrm{Cnc}$ derived in this work would imply an Alfvén surface that is larger than that we computed. In this case, an unseen axisymmetric dipolar component would only reinforce our results that $55 \mathrm{Cnc}$ e orbits inside the Alfvén surface, strengthening possible SPI.

\section{Summary and discussion}

To summarise, we have used spectropolarimetric observations of $55 \mathrm{Cnc}$ to detect the stellar magnetic field and mapped the largescale magnetic field using ZDI, finding largely a tiled dipolar field. This magnetic map was used as input for a 3D MHD simulation of the stellar wind, and the properties of the wind at the positions of the known planets were estimated. We found that $55 \mathrm{Cnc}$ e orbits entirely within the Alfvén surface of the stellar wind, which implies magnetic field lines connect planet e to the star, allowing for magnetic SPIs. Using these simulations, we estimated the possible position of chromospheric hotspots due to this interaction, and found they would be offset in phase from the planet's orbital position and that apparent activity due to SPI may be modulated with a period close to twice the orbital or synodic period. Recently, Sulis et al. (2019) detected a stellar flux modulation in phase with the orbital period of planet e. Furthermore, the amplitude of this modulation appears to be directly related to stellar activity, indicating that this could be caused by magnetic interaction occurring as a consequence of the planet lying entirely within the Alfvén surface of the star. Assuming the interpretation of the observation by Sulis et al. (2019) in terms of SPI is correct, the possible apparent discrepancy in the timing of the modulation between the observation and the model prediction may be caused by the fact that the photometric and spectropolarimetric observations have been obtained more than two years apart: within this time, the geometry and strength of the stellar magnetic field may have changed.

Ideally, contemporaneous observations of different activity proxies would be able to better characterise any signature of SPI. Additionally, future spectropolarimetric observations of the system would also allow us to investigate whether the largescale field is varying with time, and how. In particular, we would be able to further investigate the axisymmetric component, given that we found that the magnetic field is dominantly non-axisymmetric. New spectropolarimetric observations over a longer time period would also allow us to further constrain the level of differential rotation on $55 \mathrm{Cnc}$. In our analysis, it appears that differential rotation is not exceptionally strong in this star. Thanks to its high photometric precision, the CHEOPS mission will be able to follow the amplitude of the photometric modulation, which would be particularly powerful when combined with contemporaneous spectropolarimetric monitoring (e.g. Fares et al. 2017).

Acknowledgements. A.A.V., D.Ó.F., C.P.F. acknowledge joint funding received from the Irish Research Council and Campus France through the Ulysses funding scheme. The authors acknowledge the DJEI/DES/SFI/HEA Irish Centre for High-End Computing (ICHEC) for the provision of computational facilities and support. This work used the BATS-R-US tools developed at the University of Michigan Center for Space Environment Modeling and made available through the NASA Community Coordinated Modeling Center. D.Ó.F. acknowledges 
funding received from the Trinity College Postgraduate Award. D.D. acknowledges NASA Hubble Fellowship (HST-HF2-51372.001-A). A.A.V. acknowledges funding received from the Irish Research Council Laureate Awards 2017/2018 and from the European Research Council (ERC) under the European Union's Horizon 2020 research and innovation programme (grant agreement No 817540 , ASTROFLOW). J.F.D. acknowledges funding from the European Research Council (ERC) under the H2020 research \& innovation programme (grant agreement \#740651 NewWorlds).

\section{References}

Alvarado-Gómez, J. D., Drake, J. J., Cohen, O., Moschou, S. P., \& Garraffo, C. 2018, ApJ, 862, 93

Baluev, R. V. 2015, MNRAS, 446, 1493

Bhardwaj, A., \& Gladstone, G. R. 2000, Rev. Geophys., 38, 295

Bourrier, V., Dumusque, X., Dorn, C., et al. 2018, A\&A, 619, A1

Cauley, P. W., Shkolnik, E. L., Llama, J., Bourrier, V., \& Moutou, C. 2018, AJ, 156,262

Cuntz, M., \& Shkolnik, E. 2002, Astron. Nachr., 323, 387

Cuntz, M., Saar, S. H., \& Musielak, Z. E. 2000, ApJ, 533, L151

Demory, B.-O., Gillon, M., de Wit, J., et al. 2016a, Nature, 532, 207

Demory, B.-O., Gillon, M., Madhusudhan, N., \& Queloz, D. 2016b, MNRAS, 455, 2018

Donati, J.-F., Semel, M., Carter, B. D., Rees, D. E., \& Collier Cameron, A. 1997, MNRAS, 291, 658

Donati, J.-F., Howarth, I. D., Jardine, M. M., et al. 2006, MNRAS, 370, 629

Dragomir, D., Matthews, J. M., Winn, J. N., \& Rowe, J. F. 2014, IAU Symp., 293, 52

Fares, R., Donati, J. F., Moutou, C., et al. 2012, MNRAS, 423, 1006

Fares, R., Bourrier, V., Vidotto, A. A., et al. 2017, MNRAS, 471, 1246

Fischer, C., \& Saur, J. 2019, ApJ, 872, 113

Folsom, C. P., Bouvier, J., Petit, P., et al. 2018a, MNRAS, 474, 4956

Folsom, C. P., Fossati, L., Wood, B. E., et al. 2018b, MNRAS, 481, 5286

Hempelmann, A., Mittag, M., Gonzalez-Perez, J. N., et al. 2016, A\&A, 586, A14
Ip, W.-H., Kopp, A., \& Hu, J.-H. 2004, ApJ, 602, L53

Jardine, M., Vidotto, A. A., \& See, V. 2017, MNRAS, 465, L25

Kochukhov, O., Makaganiuk, V., \& Piskunov, N. 2010, A\&A, 524, A5

Krüger, H., Geissler, P., Horányi, M., et al. 2003, Geophys. Res. Lett., 30 2101

Ligi, R., Creevey, O., Mourard, D., et al. 2016, A\&A, 586, A94

Manchester, W., Vourlidas, A., Toth, G., et al. 2008, ApJ, 684, 1448

Marcy, G. W. 1984, ApJ, 276, 286

McIvor, T., Jardine, M., \& Holzwarth, V. 2006, MNRAS, 367, L1

Miller, B. P., Gallo, E., Wright, J. T., \& Dupree, A. K. 2012, ApJ, 754, 137

Miller, B. P., Gallo, E., Wright, J. T., \& Pearson, E. G. 2015, ApJ, 799, 163

Moutou, C., Donati, J. F., Lin, D., Laine, R. O., \& Hatzes, A. 2016, MNRAS, 459, 1993

Neubauer, F. M. 1980, J. Geophys. Res., 85, 1171

Ó Fionnagáin, D., \& Vidotto, A. A. 2018, MNRAS, 476, 2465

Powell, K. G., Roe, P. L., Linde, T. J., Gombosi, T. I., \& De Zeeuw, D. L. 1999, J. Comp. Phys., 154, 284

Réville, V., Brun, A. S., Strugarek, A., et al. 2015, ApJ, 814, 99

Ridden-Harper, A. R., Snellen, I. A. G., Keller, C. U., et al. 2016, A\&A, 593, A129

Saur, J., Grambusch, T., Duling, S., Neubauer, F. M., \& Simon, S. 2013, A\&A, 552, A119

Shkolnik, E., Walker, G. A. H., Bohlender, D. A., Gu, P. G., \& Kürster, M. 2005, ApJ, 622, 1075

Shkolnik, E., Bohlender, D. A., Walker, G. A. H., \& Collier Cameron, A. 2008, ApJ, 676, 628

Strugarek, A., Brun, A. S., Donati, J. F., Moutou, C., \& Réville, V. 2019, ApJ, 881,136

Sulis, S., Dragomir, D., Lendl, M., et al. 2019, A\&A, 631, A129

Tóth, G., Sokolov, I. V., Gombosi, T. I., et al. 2005, J. Geophys. Res., 110, A12226

Vidotto, A. A., Fares, R., Jardine, M., Moutou, C., \& Donati, J.-F. F. 2015, MNRAS, 449, 4117

von Braun, K., Boyajian, T. S., ten Brummelaar, T. A., et al. 2011, ApJ, 740, 49

Winn, J. N., Matthews, J. M., Dawson, R. I., et al. 2011, ApJ, 737, L18 